\title{
Correlation Measure for Interval valued Pythagorean Fuzzy Sets
}

Saji Antonia $\mathfrak{S}^{1}$ and Mohana $\mathrm{K}^{*, 2}$

Received: 20 April 2021/ Accepted: 10 June 2021/ Published online: 18 June 2021

(C)Sacred Heart Research Publications 2017

\begin{abstract}
In this paper, we study the concept of Interval Valued Pythagorean set [IVPS] with special cases of membership and non-membership degrees. In this IVPS set membership and non-membership degrees are satisfying the condition $\left(u_{A}(x)\right)^{2}+\left(\nu_{A}(x)\right)^{2} \leq 1$ instead of $u_{A}(x)+\nu_{A}(x)>1$. Here the correlation measure of IVPS set is an extension of correlation measure of Interval valued Pythagorean fuzzy set.
\end{abstract}

Key words: Interval-Valued Pythagorean Fuzzy Set, Correlation Measures.

\section{Introduction}

Fuzzy sets are initiated by L.A.Zadeh. The extension of fuzzy set (IFS) describes the non-membership grade of an membership grades under a restriction that the sum of membership and non-membership grade of an imprecise does not exceed 1.

Based on the centric method, Hanafy introduced and studied the correlation and correlation coefficient. Correlation coefficients are beneficial tools used to determine the degree of similarity between objects. The correlation coefficients in fuzzy environments have many applications in the field of medicals diagnosis and clustering etc.

\section{Preliminaries}

Definition 2.1 Let $\mathrm{X}$ be a non empty set and I the unit interval $[0,1]$. A interval valued Pythagorean set $\mathrm{A}$ and $\mathrm{B}$ of the form $A=\left\{x, u_{A}(x), v_{A}(x): x \in X\right\}$ and

\footnotetext{
${ }^{1,2}$ Department of Mathematics, Nirmala College for Women, Coimbatore.

Emails: ${ }^{1}$ sajiantonia@gmail.com, ${ }^{*, 2}$ riyaraju116@gmail.com
} 
ISSN: 2456-8686, 5(1), 2021:048-059

https://doi.org/10.26524/cm91

$B=\left\{x, u_{B}(x), v_{B}(x): x \in X\right\}$. Then the correlation coefficient of $\mathrm{A}$ and $\mathrm{B}$ is

$$
\rho(A, B)=\frac{C(A, B)}{\sqrt{C(A, A) \cdot C(B, B)}}
$$

$C(A, B)=\sum_{i=1}^{n}\left(\left(u_{A}\left(x_{i}\right)\right)^{2} \cdot\left(u_{B}\left(x_{i}\right)\right)^{2}+\left(v_{A}\left(x_{i}\right)\right)^{2} \cdot\left(v_{B}\left(x_{i}\right)\right)^{2}\right)$

$C(A, A)=\sum_{i=1}^{n}\left(\left(u_{A}\left(x_{i}\right)\right)^{2} \cdot\left(u_{A}\left(x_{i}\right)\right)^{2}+\left(v_{A}\left(x_{i}\right)\right)^{2} \cdot\left(v_{A}\left(x_{i}\right)\right)^{2}\right)$

$C(B, B)=\sum_{i=1}^{n}\left(\left(u_{B}\left(x_{i}\right)\right)^{2} \cdot\left(u_{B}\left(x_{i}\right)\right)^{2}+\left(v_{B}\left(x_{i}\right)\right)^{2} \cdot\left(v_{B}\left(x_{i}\right)\right)^{2}\right)$

Definition 2.2 Let E be an universe set. An Intuitionistic fuzzy set A on E can be defined as follows:

$A=\left\{\left\langle x, u_{A}(x), v_{A}(x)>, x \in E\right\}\right.$, where $u_{A}: E \rightarrow[0,1]$ and $v_{A}: E \rightarrow[0,1]$ such that $0=u_{A}(x)+v_{A} \leq 1$ for any $x \in E$. $u_{A}(x)$ and $v_{A}(x)$ are the degree of membership and degree of non-membership of the element $x$ respectively.

Definition 2.3 Let $\mathrm{X}$ is a non-empty set (universe). A Interval valued Pythagorean fuzzy set $\mathrm{A}$ on $\mathrm{X}$ is an object of the form $A=\left\{\left(x, u_{A}(x), v_{A}(x)\right): x \in x\right\}$, where $\left.u_{A}(x), v_{A}(x) \in[0,1], 0 \leq\left(u_{A}(x)\right)^{2}+v_{A}(x)\right)^{2} \leq 1$ for all $x$ in $\mathrm{X} . u_{A}(x)$ is the degree of membership, and $v_{A}(x)$ is the degree of non-membership.

Definition 2.4 Let $\mathrm{X}$ be a non-empty set and I the unit interval [0,1]. A Intervalvalued Pythagorean fuzzy sets $\mathrm{A}$ and $\mathrm{B}$ are of the form $A=\left\{x, u_{A}(x), v_{A}(x): x \in\right.$ $X\}$ and $B=\left\{x, u_{B}(x), v_{B}(x): x \in X\right\}$.

1) $A^{c}=\left\{x, v_{A}(x), u_{A}(B(x)): x \in X\right\}$

2) $A \cup B=\left\{\left(x, \max \left(u_{A}(x), u_{B}(x)\right), \min \left(v_{A}(x), v_{B}(x)\right)\right) ; x \in X\right\}$

3) $A \cap B=\left\{\left(x, \min \left(u_{A}(x), u_{B}(x)\right), \max \left(v_{A}(x), v_{B}(x)\right)\right) ; x \in X\right\}$

Definition 2.5 Let $\mathrm{X}$ be a non-empty set and I the unit interval [0,1]. A Interval Valued Pythagorean fuzzy set $\mathrm{S}$ is an object having the form

$A=\left\{x, u_{A}(x), v_{A}(x): x \in X\right\}$, where the functions $u_{A}: X \rightarrow[0,1]$ and

$v_{A}: X \rightarrow[0,1]$ denote respectively the degree of membership and degree of non-membership of each element $x \in X$ to the set $\mathrm{P}$ and $0 \leq\left(u_{A}(x)\right)^{2}+\left(v_{A}(x)\right)^{2} \leq 1$. 
ISSN: 2456-8686, 5(1), 2021:048-059

https://doi.org/10.26524/cm91

\section{Interval- valued Pythagorean fuzzy set}

Definition 3.1 Let $\mathrm{X}$ be a non-empty set (universe). A set $\mathrm{A}$ on $\mathrm{X}$ is an object of the form $A=\left\{x, u_{A}(x), v_{A}(x): x \in X\right\}$, where $u_{A}(x), v_{A}(x) \in[0,1], 0=u_{A}(x)+v_{A}(x) \leq$ 1 , for all $x$ in X. $u_{A}(x)$ is the degree of membership, and $v_{A}(x)$ is the degree of non-membership. Here $u_{A}(x)$ and $v_{A}(x)$ are dependent components.

Definition 3.2 Let $\mathrm{X}$ be a nonempty set and I be the unit interval $[0,1]$. Let object sets $A=\left\{x, u_{A}(x), v_{A}(x): x \in X\right\}$ and $B=\left\{x, u_{B}(x), v_{B}(x): x \in X\right\}$. Then,

1) $A^{c}=\left\{x, u_{A}(x), v_{A}(x): x \in X\right\}$

2) $A \cup B=\left\{\left(x, \max \left(u_{A}(x), u_{A}(x)\right), \min \left(v_{A}(x), v_{A}(x)\right)\right) ; x \in X\right\}$

3) $A \cap B=\left\{\left(x, \min \left(u_{A}(x), u_{A}(x)\right), \max \left(v_{A}(x), v_{A}(x)\right)\right) ; x \in X\right\}$

Proposition 3.3 The defined correlation measure between IVPS A and IVPS B satisfies the following properties

(i) $0 \rho(A, B) \leq 1$

(ii) $\rho(A, B)=1$ if and only if $\mathrm{A}=\mathrm{B}$

(iii) $\rho(A, B)=\rho(B, A)$.

Proof: (i) $0 \leq \rho(A, B) \leq 1$

As the membership, indeterminate and non-membership functions of the IVPS lies between 0 and $1, \rho(A, B)$, Also lies between 0 and 1 . We will prove

$$
\begin{gathered}
c(A, B)=\sum_{i=1}^{n}\left(u_{A}\left(x_{i}\right)\right)^{2} \cdot\left(u_{B}\left(x_{i}\right)\right)^{2}+\left(v_{A}\left(x_{i}\right)\right)^{2} \cdot\left(v_{B}\left(x_{i}\right)\right)^{2} \\
=\left(\left(u_{A}\left(x_{1}\right)\right)^{2} \cdot\left(u_{B}\left(x_{1}\right)\right)^{2}+\left(v_{A}\left(x_{1}\right)\right)^{2} \cdot\left(v_{B}\left(x_{1}\right)\right)^{2}+\left(\left(u_{A}\left(x_{2}\right)\right)^{2} \cdot\left(u_{B}\left(x_{2}\right)\right)^{2}\right.\right. \\
+\left(v_{A}\left(x_{2}\right)\right)^{2} \cdot\left(v_{B}\left(x_{2}\right)\right)^{2}+\cdots+\left(\left(u_{A}\left(x_{n}\right)\right)^{2} \cdot\left(u_{B}\left(x_{n}\right)\right)^{2}+\left(v_{A}\left(x_{n}\right)\right)^{2} \cdot\left(v_{B}\left(x_{n}\right)\right)^{2}\right)
\end{gathered}
$$

By Cauchy-Schwartz inequality

$$
\left(x_{1} y_{1}+x_{2} y_{2}+\cdots+x_{n} y_{n}\right)^{2}=\left(x_{1}^{2}+x_{2}^{2}+\cdots+x_{n}^{2}\right) \cdot\left(y_{1}^{2}+y_{2}^{2}+\cdots+y_{n}^{2}\right) \text {, }
$$

where $\left(x_{1}+x_{2}+\cdots x_{n}\right) \in R^{n}$ and $\left(y_{1}+y_{2}+\cdots y_{n}\right) \in R^{n}$,

we get $\left.(c(A, B))^{2}=\left(\left(u_{A}\left(x_{1}\right)\right)^{4}+\left(v_{A}\left(x_{1}\right)\right)^{4}\right)+\left(u_{A}\left(x_{2}\right)\right)^{4}+\left(v_{A}\left(x_{2}\right)\right)^{4}\right)$

$$
\begin{gathered}
+\cdots+\left(\left(u_{A}\left(x_{1}\right)\right)^{4}+\left(v_{A}\left(x_{n}\right)\right)^{2}\right) \times\left(\left(u_{B}\left(x_{1}\right)\right)^{4}+\left(v_{B}\left(x_{1}\right)\right)^{4}\right) \\
+\left(\left(u_{B}\left(x_{2}\right)\right)^{4}+\left(v_{B}\left(x_{n}\right)\right)^{4}\right)+\cdots+\left(\left(u_{B}\left(x_{n}\right)\right)^{4}+\left(v_{B}\left(x_{n}\right)\right)^{4}\right) \\
=\left(u_{A}\left(x_{1}\right)\right)^{2} \cdot\left(u_{A}\left(x_{1}\right)\right)^{2}\left(\left(v_{A}\left(x_{1}\right)\right)^{2} \cdot\left(v_{A}\left(x_{1}\right)\right)^{2}\right)\left(\left(u_{A}\left(x_{2}\right)\right)^{2} \cdot\left(u_{A}\left(x_{2}\right)\right)^{2}\right. \\
\left.+\left(v_{A}\left(x_{2}\right)\right)^{2} \cdot\left(v_{A}\left(x_{2}\right)\right)^{2}\right)+\cdots+\left(\left(u_{A}\left(x_{n}\right)\right)^{2} \cdot\left(u_{A}\left(x_{n}\right)\right)^{2}\right. \\
\hline
\end{gathered}
$$


ISSN: 2456-8686, 5(1), 2021:048-059

https://doi.org/10.26524/cm91

$$
\begin{aligned}
& \left.+\left(v_{A}\left(x_{n}\right)\right)^{2} \cdot\left(v_{A}\left(x_{n}\right)\right)^{2}\right) \times\left(\left(u_{B}\left(x_{1}\right)\right)^{2} \cdot\left(u_{B}\left(x_{1}\right)\right)^{2}+\left(v_{B}\left(x_{1}\right)\right)^{2} \cdot\left(v_{B}\left(x_{1}\right)\right)^{2}\right) \\
& +\left(\left(u_{B}\left(x_{2}\right)\right)^{2} \cdot\left(u_{B}\left(x_{2}\right)\right)^{2}+\left(v_{B}\left(x_{2}\right)\right)^{2} \cdot\left(v_{B}\left(x_{2}\right)\right)^{2}\right. \\
& +\cdots+\left(\left(u_{B}\left(x_{n}\right)\right)^{2} \cdot\left(\left(u_{B}\left(x_{n}\right)\right)^{2}+\left(v_{B}\left(x_{n}\right)\right)^{2} \cdot\left(v_{B}\left(x_{n}\right)\right)^{2}\right)\right.
\end{aligned}
$$

$=C(A, A) \times C(B, B)$

Therefore $(C(A, B))^{2} \leq C(A, A) \times C(B, B)$ and thus $\rho(A, B) \leq 1$. Hence we obtain the following property $0 \leq \rho(A, B) \leq 1$

(ii) $\rho(A, B)=1$ if and only if $\mathrm{A}=\mathrm{B}$

Let the two IVPS A and IVPS B be equal (i.e. $\mathrm{A}=\mathrm{B}$ ). Hence for any $u_{A}\left(x_{i}\right)=u_{B}\left(x_{i}\right)$ and $v_{A}\left(x_{i}\right)=v_{B}\left(x_{i}\right)$ Then

$$
\begin{gathered}
C(A, A)=C(B, B)=\sum_{i=1}^{n}\left(\left(u_{A}\left(x_{i}\right)\right)^{2} \cdot\left(u_{A}\left(x_{i}\right)\right)^{2}+\left(v_{A}\left(x_{i}\right)\right)^{2} \cdot\left(v_{A}\left(x_{i}\right)\right)^{2}\right) \text { and } \\
C(A, B)=\sum_{i=1}^{n}\left(\left(u_{A}\left(x_{i}\right)\right)^{2} \cdot\left(u_{B}\left(x_{i}\right)\right)^{2}+\left(v_{A}\left(x_{i}\right)\right)^{2} \cdot\left(v_{B}\left(x_{i}\right)\right)^{2}\right. \\
=\sum_{i=1}^{n}\left(u_{A}\left(x_{i}\right)\right)^{2} \cdot\left(u_{A}\left(x_{i}\right)\right)^{2}+\left(v_{A}\left(x_{i}\right)\right)^{2} \cdot\left(v_{A}\left(x_{i}\right)\right)^{2}=C(A, A)
\end{gathered}
$$

Hence, $\rho(A, B)=\frac{C(A, B)}{\sqrt{C(A, A) \cdot C(B, B)}}=\frac{C(A, A)}{\sqrt{C(A, A) \cdot C(A, A)}}=1$.

Let the $\rho(A, B)=1$. Then, the unite measure is possible only if $\frac{C(A, B)}{\sqrt{C(A, A) \cdot C(B, B)}}=1$. This refer that $u_{A}\left(x_{i}\right)=u_{B}\left(x_{i}\right)$ and $v_{A}\left(x_{i}\right)=v_{B}\left(x_{i}\right)$ for all i $\mathrm{A}=\mathrm{B}$

iii) If $\rho(A, B)=\rho(B, A)$, it obvious that

$$
\begin{gathered}
\frac{C(A, B)}{\sqrt{C(A, A) \cdot C(B, B)}}=\frac{C(A, B)}{\sqrt{C(A, A) \cdot C(B, B)}}=\rho(B, A) \\
C(A, B)=\sum_{i=1}^{n}\left(\left(u_{A}\left(x_{i}\right)\right)^{2} \cdot\left(u_{B}\left(x_{i}\right)\right)^{2}+\left(v_{A}\left(x_{i}\right)\right)^{2} \cdot\left(v_{A}\left(x_{i}\right)\right)^{2}\right) \\
=\sum_{i=1}^{n}\left(\left(u_{A}\left(x_{i}\right)\right)^{2} \cdot\left(u_{A}\left(x_{i}\right)\right)^{2}+\left(v_{B}\left(x_{i}\right)\right)^{2} \cdot\left(v_{A}\left(x_{i}\right)\right)^{2}\right)=C(B, A),
\end{gathered}
$$

which completes the proof. 
ISSN: 2456-8686, 5(1), 2021:048-059

https://doi.org/10.26524/cm91

Definition 3.4 Let A and B be two IVPS. The correlation coefficient is defined as

$$
\rho^{\prime}=\frac{C(A, B)}{\max \{C(A, A) \cdot C(B, B)\}}
$$

Theorem 3.5 The defined correlation measure between IVPS A and IVPS B satisfies the following properties

i) $0 \leq \rho^{\prime}(A, B) \leq 1$

ii) $\rho^{\prime}(A, B)=1$ if and only if $\mathrm{A}=\mathrm{B}$

iii) $\rho^{\prime}(A, B)=\rho^{\prime}(B, A)$

Proof: The property is straight forward, so omit here. Also $\rho^{\prime}(A, B) \geq 0$ is evident. We now prove only $\rho^{\prime}(A, B) \leq 1$. Since $\left((C(A, B))^{2} \leq C(A, A) \cdot C(B, B)\right.$ we have $C(A, B) \leq \max \{C(A, A), C(B, B)\}$ and thus $\rho^{\prime}(A, B) \leq 1$. However, in many practical situations, the different set may have taken different weights and weight $\omega_{i}$ of the element $x_{i} \in X(i=1,2, \cdots n)$ should be taken into account. In the following, we develop a weighted correlation coefficient between IVPS.

Let $\omega=\left\{\omega_{1}, \omega_{2}, \cdots \omega_{n}\right\}$ be the weight vector of the elements $x_{i}(i=1,2, . ., n)$ with $\omega_{i} \geq 0$ and $\sum_{i=1}^{n} \omega_{i}=1$, then we have extended the above correlation coefficient $\rho(A, B)$ and $\rho^{\prime}(A, B)$ to weighted correlation coefficient as follows:

$$
\begin{gathered}
\rho^{\prime \prime}=\frac{C_{\omega}(A, B)}{\sqrt{C_{\omega}(A, A) \cdot C_{\omega}(B, B)}} \\
C_{\omega}(A, B)=\sum_{i=1}^{n} \omega_{i}\left(\left(u_{A}\left(x_{i}\right)\right)^{2} \cdot\left(u_{B}\left(x_{i}\right)\right)^{2}+\left(v_{A}\left(x_{i}\right)\right)^{2} \cdot\left(v_{B}\left(x_{i}\right)\right)^{2}\right. \\
C_{\omega}(A, A)=\sum_{i=1}^{n} \omega_{i}\left(\left(u_{A}\left(x_{i}\right)\right)^{2} \cdot\left(u_{A}\left(x_{i}\right)\right)^{2}+\left(v_{A}\left(x_{i}\right)\right)^{2} \cdot\left(v_{A}\left(x_{i}\right)\right)^{2}\right. \\
C_{\omega}(B, B)=\sum_{i=1}^{n} \omega_{i}\left(\left(u_{B}\left(x_{i}\right)\right)^{2} \cdot\left(u_{B}\left(x_{i}\right)\right)^{2}+\left(v_{B}\left(x_{i}\right)\right)^{2} \cdot\left(v_{B}\left(x_{i}\right)\right)^{2}\right.
\end{gathered}
$$

and

$$
\rho^{\prime \prime}=\frac{C_{\omega}(A, B)}{\max \left\{C_{\omega}(A, A) \cdot C_{\omega}(B, B)\right\}}
$$


ISSN: 2456-8686, 5(1), 2021:048-059

https://doi.org/10.26524/cm91

$$
=\frac{\sum_{i=1}^{n} \omega_{i}\left(\left(u_{A}\left(x_{i}\right)\right)^{2} \cdot\left(u_{B}\left(x_{i}\right)\right)^{2}+\left(v_{A}\left(x_{i}\right)\right)^{2} \cdot\left(v_{B}\left(x_{i}\right)\right)^{2}\right)}{\max \left\{\begin{array}{c}
\sum_{i=1}^{n} \omega_{i}\left(\left(u_{A}\left(x_{i}\right)\right)^{2} \cdot\left(u_{A}\left(x_{i}\right)\right)^{2}+\left(v_{A}\left(x_{i}\right)\right)^{2} \cdot\left(v_{A}\left(x_{i}\right)\right)^{2}\right. \\
\sum_{i=1}^{n} \omega_{i}\left(\left(u_{B}\left(x_{i}\right)\right)^{2} \cdot\left(u_{B}\left(x_{i}\right)\right)^{2}+\left(v_{B}\left(x_{i}\right)\right)^{2} \cdot\left(v_{B}\left(x_{i}\right)\right)^{2}\right.
\end{array}\right\}}
$$

It is easy to verify that if $\omega=\left(\frac{1}{n}, \frac{1}{n}, \ldots, \frac{1}{n}\right)^{T}$, then equations (3) and (4) reduces to (1) and (2) respectively.

Theorem 3.6 Let $\omega=\left(\omega_{1}, \omega_{2}, \cdots, \omega_{n}\right)^{T}$ be the weight vector of $x_{i}(i=1,2 . . n)$ with $\omega_{i} \geq 0$ and $\sum_{i=1}^{n} \omega_{i}=1$,then the weighted correlation coefficient between IVPS A and IVPS B satisfies:

i) $0 \leq \rho^{\prime \prime}(A, B) \leq 1$

ii) $\rho^{\prime \prime}(A, B)=1$ if and only if $\mathrm{A}=\mathrm{B}$

iii) $\rho^{\prime \prime}(A, B)=\rho^{\prime \prime}(B, A)$

Proof: The property (i) and (ii) are straight forward.Also $\rho^{\prime \prime}(A, B) \geq 0$ is evident so we need to show $\rho^{\prime \prime}(A, B) \leq 1$. Since,

$$
\begin{aligned}
& C_{\omega}(A, B)=\sum_{i=1}^{n} \omega_{i}\left(\left(u_{A}\left(x_{i}\right)\right)^{2} \cdot\left(u_{B}\left(x_{i}\right)\right)^{2}+\left(v_{A}\left(x_{i}\right)\right)^{2} \cdot\left(v_{B}\left(x_{i}\right)\right)^{2}\right) \\
& =\omega_{1}\left(\left(u_{A}\left(x_{1}\right)\right)^{2} \cdot\left(u_{B}\left(x_{1}\right)\right)^{2}+\left(v_{A}\left(x_{1}\right)\right)^{2} \cdot\left(v_{B}\left(x_{1}\right)+\omega_{2}\left(u_{A}\left(x_{2}\right)\right)^{2} \cdot\left(u_{B}\left(x_{2}\right)\right)^{2}\right.\right. \\
& +\left(v_{A}\left(x_{2}\right)\right)^{2} \cdot\left(v_{B}\left(x_{2}\right)\right)^{2}+\cdots+\omega_{n}\left(\left(u_{A}\left(x_{n}\right)\right)^{2} \cdot\left(u_{B}\left(x_{n}\right)\right)^{2}+\left(v_{A}\left(x_{n}\right)\right)^{2} \cdot\left(v_{B}\left(x_{n}\right)\right)^{2}\right. \\
& \left.=\left(\sqrt{\omega_{1}(} u_{A}\left(x_{1}\right)\right)^{2} \cdot \sqrt{\omega_{1}}\left(u_{B}\left(x_{1}\right)\right)^{2}+\sqrt{\omega_{1}}\left(v_{A}\left(x_{1}\right)\right)^{2} \cdot \sqrt{\omega_{1}}\left(v_{B}\left(x_{1}\right)\right)^{2}\right) \\
& \quad+\left(\sqrt{\omega_{2}}\left(u_{A}\left(x_{2}\right)\right)^{2} \cdot \sqrt{\omega_{2}}\left(u_{B}\left(x_{2}\right)\right)^{2}\left(\sqrt{\omega_{2}}\left(v_{A}\left(x_{2}\right)\right)^{2} \cdot \sqrt{\omega_{2}}\left(v_{B}\left(x_{2}\right)\right)^{2}\right)\right. \\
& \left.\quad+. .+\left(\sqrt{\omega_{n}}\left(u_{A}\left(x_{n}\right)\right)^{2} \cdot \sqrt{\omega_{n}(} u_{B}\left(x_{n}\right)\right)^{2}+\sqrt{\omega_{n}}\left(v_{A}\left(x_{n}\right)\right)^{2} \cdot \sqrt{\omega_{n}}\left(v_{B}\left(x_{n}\right)\right)^{2}\right)
\end{aligned}
$$

By using Cauchy-Schwarz inequality, we get

$$
\begin{aligned}
&\left.C_{w}(A, B)\right)^{2}=\left(w_{1}\left(u_{A}\left(x_{1}\right)\right)^{2} \cdot\left(u_{A}\left(x_{1}\right)\right)^{2}+\left(v_{A}\left(x_{1}\right)\right)^{2} \cdot\left(v_{A}\left(x_{1}\right)\right)^{2}\right) \\
&+\left(w_{2}\left(u_{A}\left(x_{2}\right)\right)^{2} \cdot\left(u_{A}\left(x_{2}\right)\right)^{2}+\left(v_{A}\left(x_{2}\right)\right)^{2} \cdot\left(v_{A}\left(x_{2}\right)\right)^{2}\right) \\
&+\cdots+\left(w_{n}\left(u_{A}\left(x_{n}\right)\right)^{2} \cdot\left(u_{A}\left(x_{n}\right)\right)^{2}\right. \\
&\left.+\left(v_{A}\left(x_{n}\right)\right)^{2} \cdot\left(v_{A}\left(x_{n}\right)\right)^{2}\right) \times\left(w_{1}\left(u_{B}\left(x_{1}\right)\right)^{2} \cdot\left(u_{B}\left(x_{1}\right)\right)^{2}\right. \\
&\left.+\left(v_{B}\left(x_{1}\right)\right)^{2} \cdot\left(v_{B}\left(x_{1}\right)\right)^{2}\right)+\left(w_{2}\left(u_{B}\left(x_{2}\right)\right)^{2} \cdot\left(u_{B}\left(x_{2}\right)\right)^{2}+\left(v_{B}\left(x_{2}\right)\right)^{2} \cdot\left(v_{B}\left(x_{2}\right)\right)^{2}\right)
\end{aligned}
$$


ISSN: 2456-8686, 5(1), 2021:048-059

https://doi.org/10.26524/cm91

$$
\begin{gathered}
+\cdots+\left(w_{n}\left(u_{B}\left(x_{n}\right)\right)^{2} \cdot\left(u_{B}\left(x_{n}\right)\right)^{2}+\left(v_{B}\left(x_{n}\right)\right)^{2} \cdot\left(v_{B}\left(x_{n}\right)\right)^{2}\right) \\
=\sum_{i=1}^{n} w_{i}\left(\left(u_{A}\left(x_{i}\right)\right)^{2} \cdot\left(u_{A}\left(x_{i}\right)\right)^{2}+\left(v_{A}\left(x_{i}\right)\right)^{2}\left(v_{A}\left(x_{i}\right)\right)^{2}\right. \\
\times \sum_{i=1}^{n} w_{i}\left(\left(u_{B}\left(x_{i}\right)\right)^{2} \cdot\left(u_{B}\left(x_{i}\right)\right)^{2}+\left(v_{B}\left(x_{i}\right)\right)^{2}\left(v_{B}\left(x_{i}\right)\right)^{2}\right. \\
=C_{\omega}(A, A) \times C_{\omega}(B, B)
\end{gathered}
$$

Therefore $C_{\omega}(A, B) \leq \sqrt{C_{\omega}(A, A) \times C_{\omega}(B, B)}$ and hence $0 \leq \rho^{\prime \prime}(A, B) \leq 1$.

Theorem 3.7 The correlation coefficient of IVPS A and IVPS B defined in $\rho^{\prime \prime}(A, B)$ satisfies the properties as given in Theorem 3.6.

Proof: $\rho(A, B)$ and $\rho^{\prime}(A, B)$ to weighted correlation coefficient as follows:

$$
\begin{gathered}
\rho^{\prime \prime}=\frac{C_{\omega}(A, B)}{\sqrt{C_{\omega}(A, A) \cdot C_{\omega}(B, B)}} \\
C_{\omega}(A, B)=\sum_{i=1}^{n} \omega_{i}\left(\left(u_{A}\left(x_{i}\right)\right)^{2} \cdot\left(u_{B}\left(x_{i}\right)\right)^{2}+\left(v_{A}\left(x_{i}\right)\right)^{2} \cdot\left(v_{B}\left(x_{i}\right)\right)^{2}\right. \\
C_{\omega}(A, A)=\sum_{i=1}^{n} \omega_{i}\left(\left(u_{A}\left(x_{i}\right)\right)^{2} \cdot\left(u_{A}\left(x_{i}\right)\right)^{2}+\left(v_{A}\left(x_{i}\right)\right)^{2} \cdot\left(v_{A}\left(x_{i}\right)\right)^{2}\right. \\
C_{\omega}(B, B)=\sum_{i=1}^{n} \omega_{i}\left(\left(u_{B}\left(x_{i}\right)\right)^{2} \cdot\left(u_{B}\left(x_{i}\right)\right)^{2}+\left(v_{B}\left(x_{i}\right)\right)^{2} \cdot\left(v_{B}\left(x_{i}\right)\right)^{2}\right.
\end{gathered}
$$

And

$$
\begin{aligned}
& \rho^{\prime \prime}=\frac{C_{\omega}(A, B)}{\max \left\{C_{\omega}(A, A) \cdot C_{\omega}(B, B)\right\}} \\
& =\frac{\sum_{i=1}^{n} \omega_{i}\left(\left(u_{A}\left(x_{i}\right)\right)^{2} \cdot\left(u_{B}\left(x_{i}\right)\right)^{2}+\left(v_{A}\left(x_{i}\right)\right)^{2} \cdot\left(v_{B}\left(x_{i}\right)\right)^{2}\right)}{\max \left\{\begin{array}{l}
\sum_{i=1}^{n} \omega_{i}\left(\left(u_{A}\left(x_{i}\right)\right)^{2} \cdot\left(u_{A}\left(x_{i}\right)\right)^{2}+\left(v_{A}\left(x_{i}\right)\right)^{2} \cdot\left(v_{A}\left(x_{i}\right)\right)^{2}\right. \\
\sum_{i=1}^{n} \omega_{i}\left(\left(u_{B}\left(x_{i}\right)\right)^{2} \cdot\left(u_{B}\left(x_{i}\right)\right)^{2}+\left(v_{B}\left(x_{i}\right)\right)^{2} \cdot\left(v_{B}\left(x_{i}\right)\right)^{2}\right.
\end{array}\right\}}
\end{aligned}
$$

It is easy to verify that if $\omega=\left(\frac{1}{n}, \frac{1}{n}, \cdots, \frac{1}{n}\right)^{T}$, then equation (6) and (5) reduces (1) and (2) respectively.

Example 3.8 Let $\mathrm{P}=\left\{P_{1}, P_{2}, P_{3}\right\}$ be a set of patients, $\mathrm{D}=\{$ Viral Fever,Malaria,Typoid,Dengu $\}$ be a set of diseases and $\mathrm{S}=\{$ Tempreaure,Headache,Cough,Joint Pain $\}$ be a set of symptoms 
ISSN: 2456-8686, 5(1), 2021:048-059

https://doi.org/10.26524/cm91

Table 1:M (the relation between Patient and Symptoms)

\begin{tabular}{|c|c|c|c|c|}
\hline $\mathrm{M}$ & Temperature & Headache & Cough & Joint Pain \\
\hline$P_{1}$ & $(0.7,0.6) ;(0.5,0.7)$ & $(0.4,0.6) ;(0.7,0.7)$ & $(0.5,0.7) ;(0.3,0.5)$ & $(0.2,0.4) ;(0.1,0.3)$ \\
\hline$P_{2}$ & $(0.1,0.3) ;(0.8,0.9)$ & $(0.4,0.6) ;(0.7,0.7)$ & $(0.3,0.5) ;(0.2,0.4)$ & $(0.1,0.2) ;(0.8,0.9)$ \\
\hline$P_{3}$ & $(0.2,0.4) ;(0.4,0.6)$ & $(0.7,0.8) ;(0.5,0.5)$ & $(0.3,0.5) ;(0.8,0.8)$ & $(0.4,0.6) ;(0.1,0.3)$ \\
\hline
\end{tabular}

Table 2:N( the relation between Symptoms and Diseases)

\begin{tabular}{|c|c|c|c|c|}
\hline $\mathrm{N}$ & Viral Fever & Malaria & Typhoid & Dengu \\
\hline Temperature & $(0.8,0.7) ;(0.3,0.5)$ & $(0.4,0.6) ;(0.5,0.7)$ & $(0.7,0.8) ;(0.3,0.5)$ & $(0.1,0.3) ;(0.4,0.6)$ \\
\hline Headache & $(0.1,0.2) ;(0.2,0.4)$ & $(0.4,0.6) ;(0.6,0.8)$ & $(0.3,0.4) ;(0.8,0.8)$ & $(0.8,0.9) ;(0.2,0.4)$ \\
\hline Cough & $(0.2,0.4) ;(0.7,0.9)$ & $(0.8,0.8) ;(0.3,0.5)$ & $(0.1,0.2) ;(0.8,0.9)$ & $(0.4,0.5) ;(0.7,0.7)$ \\
\hline Joint Paint & $(0.6,0.8) ;(0.4,0.5)$ & $(07,0.8) ;(0.5,0.6)$ & $(0.4,0.6) ;(0.5,0.7)$ & $(0.1,0.2) ;(0.7,0.9)$ \\
\hline
\end{tabular}

Using Equations (1), we get the value of $(\mathrm{A}, \mathrm{B})$

Table 3: $M$ and $N$ (Correlation Measure)

\begin{tabular}{|c|c|c|c|c|}
\hline $\mathrm{M}$ & Viral Fever & Malaria & Typhoid & Dengu \\
\hline$P_{1}$ & 0.5089 & $\mathbf{0 . 7 8 1 0 6}$ & $\mathbf{0 . 8 2 5 6}$ & 0.5363 \\
\hline$P_{2}$ & 0.3745 & 0.6540 & 0.6112 & $\mathbf{0 . 7 3 8 3}$ \\
\hline$P_{3}$ & $\mathbf{0 . 6 7 5 4 0}$ & 0.6582 & 0.8023 & 0.7721 \\
\hline
\end{tabular}

Using Equations (2), we get the value of $\rho^{\prime}(A, B)$

Table 4: M and N (Correlation Measure) 
ISSN: 2456-8686, 5(1), 2021:048-059

https://doi.org/10.26524/cm91

\begin{tabular}{|c|c|c|c|c|}
\hline $\mathrm{M}$ & Viral Fever & Malaria & Typhoid & Dengu \\
\hline$P_{1}$ & $\mathbf{0 . 4 3 9 6}$ & 0.78106 & 0.8256 & 0.5363 \\
\hline$P_{2}$ & 0.3745 & $\mathbf{0 . 6 5 4 0}$ & 0.6112 & 0.7383 \\
\hline$P_{3}$ & 0.67540 & 0.6582 & 0.8023 & $\boldsymbol{0 . 7 7 2 1}$ \\
\hline
\end{tabular}

On the other hand, if we assign weights $0.10,0.20,0.30$ and 0.40 respectively, then by applying correlation coefficient given in equations (3) and (4), we can give the following values of the correlation coefficient:

Using Equations (3), we get the value of $\rho^{\prime \prime}(A, B)$.

\section{Table 5: $M$ and $N$ (Correlation Measure)}

\begin{tabular}{|l|l|l|l|l|}
\hline $\mathrm{M}$ & Viral Fever & Malaria & Typhoid & Dengu \\
\hline$P_{1}$ & 0.55205 & 05826 & 0.6760 & 0.5180 \\
\hline$P_{2}$ & 0.3169 & $\mathbf{O . 6 2 2 2}$ & 0.35476 & $\boldsymbol{0 . 7 9 7 7}$ \\
\hline$P_{3}$ & $\mathbf{0 . 7 5 5 0}$ & 0.704508 & $\mathbf{0 . 7 2 6 3 2}$ & 0.78140 \\
\hline
\end{tabular}

Using Equations (4), we get the value of $\rho^{\prime \prime}(A, B)$.

\section{Table 6: $M$ and $N$ (Correlation Measure)}

\begin{tabular}{|l|l|l|l|l|}
\hline $\mathrm{M}$ & Viral Fever & Malaria & Typhoid & Dengu \\
\hline$P_{1}$ & 0.4014 & 0.4405 & 0.4427 & 0.33477 \\
\hline$P_{2}$ & 0.2967 & $\mathbf{0 . 5 8 2 6}$ & 0.3544 & 0.7581 \\
\hline$P_{3}$ & 0.71321 & 0.55140 & 0.70868 & $\mathbf{0 . 7 3 4 1 7}$ \\
\hline
\end{tabular}

The highest correlation measure from the Tables 3, 4, 5 and 6 give the proper medical diagnosis. Therefore, patient $P_{1}$ suffers from Viral Fever, patient $P_{2}$ suffers from Malaria and patient $P_{3}$ suffers from Dengu. Hence, we can see from the above four kinds of correlation coefficient indices that the results are same. 
ISSN: 2456-8686, 5(1), 2021:048-059

https://doi.org/10.26524/cm91

\section{Conclusion}

In this paper, we found the correlation measure of Interval valued Pythagorean set [IVPS] and proved some of their basic properties. Based on that the present paper, we have extended the theory of correlation coefficient to the Pythagorean in which the constraint condition of sum of membership, non-membership and indeterminacy be less. Illustrate examples have handle the situation where the existing correlation coefficient in NS environment fails. Also to deal with the situations where the elements in a set are correlative, a weighted correlation coefficients has been defined. We studied an application of correlation measure of Interval valued Pythagorean set.

\section{References}

[1] Abdel-Basset M, El-hoseny M, Gamal A, Smarandache F, A Novel Model for Evaluation Hospital Medical Care Systems Based on Plithogenic Sets, Artificial Intelligence in Medicine, 101710.

[2] Abdel-Basset M, Mohamed R , Zaied A E N H, Smarandache F, A Hybrid Plithogenic decision-making approach with quality function deployment for selecting supply chain sustainabilitymetrics, Symmetry, 11, 903 (2019).

[3] Abdel-Basset M, Manogaran G, Gamal A, Chang V, A Novel Intelligent Medical Decision Support Model Based on Soft Computing and IoT, IEEE Internet Things Journal, 2019.

[4] Abdel-Basset M, Nabeeh N A, El-Ghareeb H A, Aboelfetouh A, Utilising neutrosophic theory to solve transition difficulties of IoT-based enterprises, Enterprise Information Systems, 1-21, 2019.

[5] Abdel-Basset M, Saleh M, Gamal A, Smarandache F, An approach of TOPSIS techniquefor developing supplier selection with group decision making under type-2 neutrosophic number, Applied Soft Computing, 77, 438-452 (2019).

[6] Atanassov K ,Intuitionistic Fuzzy Sets, Fuzzy Sets and Systems, 20, 87-96 (1986).

[7] Atanassov K and Gargov G, Interval-Valued Intuitionistic Fuzzy Sets, Fuzzy Sets and Systems, 31, 343- 349 (1989).

[8] Atanassov K, Norms and Metrics Over Intuitionistic Fuzzy Sets,BUSEFAL, 55,11-20 (1993). 
ISSN: 2456-8686, 5(1), 2021:048-059

https://doi.org/10.26524/cm91

[9] Atanassov K, Intuitionistic Fuzzy Sets,Theory and Applications, Heidelberg:Physica-Verlag, 1999.

[10] Broumi S, Deli I and Smarandache F, Relations on Interval Valued Neutrosophic Soft Sets, Journal of New Results in Science, 5, 1-20 (2014).

[11] Broumi S, Deli I, Smarandache F , Neutrosophic Parametrized Soft Set theory and its decision making problem, International Frontier Science Letters, 1, 01-11, (2014).

[12] Nabeeh N A, Abdel-Basset M, El-Ghareeb H A, Aboelfettouh A, Neutrosophic multi-criteria decision making approach for iot-based enterprises, IEEE Access, 7, 59559-59574 (2019).

[13] Kharal A, A Neutrosophic Multicriteria Decision Making Method, New Mathematics and Natural Computation, Creighton University, USA, 2013.

[14] Rajarajeswari P and Uma N, Zhang and Fu's Similarity Measure on Intuitionistic Fuzzy Multi Sets, International Journal of Innovative Research in Science, Engineering and Technology, 3, 12309-12317, (2014).

[15] Smarandache F, A Unifying Field in Logics: Neutrosophic Logic, Neutrosophy, Neutrosophic Set, Neutrosophic Probability; American Research Press: Rehoboth,DE,USA, 1999.

[16] Wang H, Smarandache F, Sunderraman R, Single-valued neutrosophic sets. Rev. Air Force Acad., 17, 10-13 (2013).

[17] Xindong peng, Yong Yan*, Some Results for Pythagorean Fuzzy Sets, International Journal of Intelligent Systems, 30, 1133-1160 (2015).

[18] Xu ZS, Zhang XL, Hesitant fuzzy multi-attribute decision making based on TOPSIS with incomplete weight information, Knowl-Based Syst, 52, 53-64 (2013).

[19] Xu ZS and Yager R R, Some Geometric Aggregation Operators Based on Intuitionistic Fuzzy Sets, International Journal of General System, 35, 417-433 (2006).

[20] Yager R R ,Pythagorean Membership Grades in Multicriteria Decision Making,IEEE Trans.Fuzzy Syst., 22, 958-965 (2014). 
ISSN: 2456-8686, 5(1), 2021:048-059

https://doi.org/10.26524/cm91

[21] Yager R R ,On Ordered Weighted Averaging Aggregation Operators in Multi-criteria Decision Making, IEEE Transactions on Systems, Man and Cybernetics, 18, 183-190 (1988).

[22] Yager R R, Abbasov A M,Pythagorean Membership Grades ,Complex Numbers and Decision Making, International Journal of Intelligent Systems, 28, 436-452 (2013).

[23] Yager R R, Pythagorean Fuzzy Subsets, In:Proc Joint IFSA World Congress and NAFIPS Annual Meeting, Edmonton, Canada, 57-61 (2013).

[24] Ye J, Multicriteria decision-making method using the correlation coefficient under single-valued neutrosophic environment, International Journal of General Systems, 42(4), 386-394 (2013).

[25] Ye S, Ye J , Dice Similarity Measure between Single Valued Neutrosophic Multisets and Its Application in Medical Diagnosis, Neutrosophic Sets and Systems, 6, 48-53 (2014). 